\section{俩 Heighten Science P U B L I C I T I O N S Corporation ISSN 2576-9537}

*Address for Correspondence: Khalayleh Harbi, General surgery department, Kaplan medical center, POB 1, Rehovot, Israel, Email: kraceve30@hotmail.com, harbikh@clalit.org.il

Submitted: 06 September 2017

Approved: 14 September 2017

Published: 15 September 2017

Copyright: @ 2017 Imam A, et al. This is an open access article distributed under the Creative Commons Attribution License, which permits unrestricted use, distribution, and reproduction in any medium, provided the original work is properly cited.

Keywords: Single anastomosis gastric bypass: Dieulafoy`s lesion; Gastrointestinal haemorrhage; Gastrectomy; Intra-operative complications

\title{
Dieulafoy's Lesion related massive Intraoperative Gastrointestinal Bleeding during single Anastomosis Gastric Bypass necessitating total Gastrectomy: A Case Report
}

\author{
Ashraf Imam, Khalayleh Harbi*, Miller Rafael, Khoury Deeb, \\ Buyeviz Victor, Guy Pines and Sapojnikov Shimon
}

Department of Surgery, Kaplan Medical Center, Rehovot, The Hebrew University, Medical School, Jerusalem, Isreal

\section{Abstract}

Introduction: Immediate postoperative gastrointestinal bleeding following bariatric bypass surgery is a major complication, and usually results from staple line hemorrhage or conventional gastro-esophageal causes. Dieulafoy's lesion is a rare cause of gastrointestinal bleeding and is usually managed by endoscopic means. Herein we present a case of massive intraoperative bleeding resulting from gastric Dieulafoy`s lesion single anastomosis gastric bypass surgery necessitating resection of the gastric pouch. This is the first description of this complication, and the difference of such a lesion from the sporadic ones is discussed.

Discussion: Gastric bypass surgery is an effective procedure for morbid obesity. The approach we have adopted for massive upper GI hemorrhage in the immediate postoperative period should be distinguished from delayed bleeding after gastric bypass. In these latter cases, marginal ulceration is more common than bleeding from the remnant gastric pouch. It is also likely that bleeding from a Dieulafoy's lesion following gastric bypass surgery represents a different disease compared to other Dieulafoy`s cases.

Conclusion: This is the first description of an intraoperative Dieulafoy's lesion bleeding during the conduct of a single anastomosis gastric bypass procedure which required gastric pouch resection. Such a lesion differs from sporadic Dieulafoy's cases, and must be considered in every case of intraoperative bleeding during gastric bypass.

\section{Introduction}

Dieulafoy`s lesion is a rare cause of massive obscure gastrointestinal haemorrhage, (defined as overt or occult bleeding from a source which cannot be readily identified by standard investigations). It represents bleeding from an essentially normal histological artery which remains abnormally large and which fails to taper within the wall of the gastrointestinal tract, running a tortuous course within the submucosa and protruding as a small defect through an area of apparently normal mucosa [1]. Typically presenting in older patients, it is believed to be an acquired phenomenon which was originally described separately by Gallard in 1884 (who initially labelled it as miliary aneurysm of the stomach) and by Georges Dieulafoy's who believed it to be an early form of peptic ulceration which he called an exculceratio simplex [2,3]. Delayed bleeding from gastric Dieulafoy`s lesion has been previously described following Roux-en-Y gastric bypass for morbid obesity [4-6]. In this setting, the differential diagnosis includes early anastomotic marginal ulceration, staple line haemorrhage and even late erosive haemorrhage originating from outside the stomach [7]. 
Herein we present a case of massive intraoperative upper gastrointestinal (GI) bleeding origination from a gastric Dieulafoy`s lesion during single anastomosis gastric bypass surgery for morbid obesity. As far as we are aware this is the first reported case of bleeding from an intraoperatively recognized Dieulafoy`s lesion during the conduct of GI surgery.

\section{Case Report}

A 54-year-old female with a body mass index of $54 \mathrm{Kg} / \mathrm{m}^{2}$, underwent a single anastomosis gastric bypass surgery using standard technique [8]. Her past medical history included non-insulin dependent diabetes mellitus and hyperlipidemia. A comprehensive preoperative evaluation was conducted including an upper GI contrast study, abdominal ultrasonography, a chest x-ray, a cardiac stress test, pulmonary function studies and standard blood testing. A routine preoperative esophago-gastroduodenoscopy (EGD) was not performed.

During the operation, the gastric pouch was constructed, and gastrotomy made for future anastomosis. At this point, bleeding was evident emerging from the pouch. Under-running of the entire staple line from the lower end of the pouch up to the esophagogastric junction including the stapled anastomotic line initially appeared successful and the gastro-jejunal anastomosis was constructed 200 centimeters $(\mathrm{cm})$ distal to the ligament of Treitz using linear stapler.

The patient remained hemodynamically stable and extubated and the end of the procedure. A few minutes following extubation the patient had a large bloody emesis. The patient was re-intubated, and EGD was performed revealing a large amount of intragastric blood and clots, precluding identification of the precise site of bleeding. The patient was re-prepped and draped, and a laparoscopy was performed revealing an extremely dilated gastric remnant which was drained initially by needle aspiration. Using a combined laparoscopic-endoscopic technique and clamping of the both afferent and efferent loops of the gastric pouch, it was evident that there was an arterial bleeding point in the posterior wall of the lesser curvature close to the esophagogastric junction. Several laparoscopic sutures over the suture lines and endoscopic clips with coagulation appeared to control the site of hemorrhage.

At this point the patient had already received 6 units of packed red blood cells, 6 units of fresh frozen plasma and 6 units of platelets with Cryoprecipitate and $500 \mathrm{mg}$ of intravenous Tranexamic Acid (Hexacapron, Teva Pharmaceuticals, Petach Tikvah, Israel). The patient developed hypothermia $\left(34^{\circ} \mathrm{C}\right)$. She was transferred to the postanesthesia care unit for further resuscitation. Shortly after arriving at the unit, a large amount of fresh blood was drained from the nasogastric (NG) tube and from her mouth. She was retransferred back to the operating suite where a laparotomy was performed via bilateral subcostal (Chevron) incision, a total gastrectomy was performed, resecting both gastric pouch and remnant stomach, converting the previous gastro-jejunostomy to a Roux-en-Y esophago-jejunostomy bypass. The biliary-pancreatic limb was $200 \mathrm{~cm}$ in length with the alimentary limb $40 \mathrm{~cm}$ from the esophagus. A feeding jeujenostomy was fashioned $30 \mathrm{~cm}$ distal to the ligament of Treitz. The patient was then transferred to the intensive care unit (ICU) for recovery. Her postoperative course was uneventful except for mild pneumonia, and she was discharged on the $12^{\text {th }}$ postoperative day. The patient is now 6 month from surgery and eating regular diet without dysphagia. Histopathology of the stomach confirmed the typical appearances of a Dieulafoy`s lesion with a prominent thickened submucosal artery which had recently bled in the gastric pouch (Figure 1).

\section{Discussion}

This unusual case represents intraoperative bleeding from a Dieulafoy`s lesion. These lesion are rarely detected during routine EGD $[5,9]$, and the test would have 


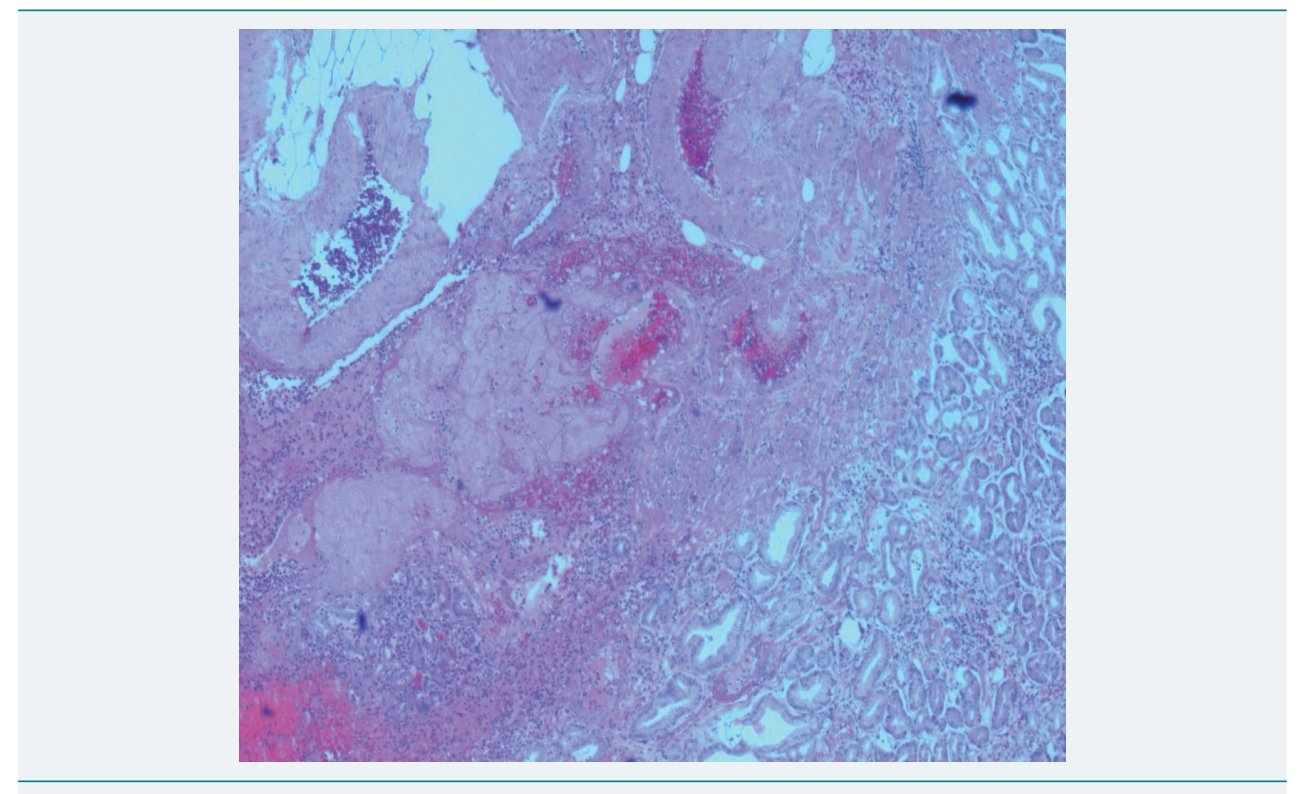

Figure 1: Histopathology of the stomach showing typical appearances of a Dieulafoy`s lesion with a prominent thickened submucosal artery which had recently bled in the gastric pouch.

probably been negative in the presented case during pre-operative EGD. The management of massive upper GI bleeding immediately following a bariatric procedure is controversial. In a super-obese patient such as the one presented, laparoscopy is recommended followed by intra-operative EGD. This allows clamping the gastric pouch to prevent over distension of the distal bowel which can obscure the view if further laparoscopic intervention is warranted [10]. The patient can then usually be managed by a combination of laparoscopicendoscopic approach where a variety of procedures can be performed depending upon the operative circumstances and surgical expertise. This can range from a simple gastrotomy with direct suturing of the bleeding vessel, facilitated by placing an endoscopic clip, and identified the exact location using endosopic trans-illumination, up to pouch gastrectomy and re-anastomosis [11]. In the presented case, the gastric pouch was fashioned over a 38Fr bougie, which results in a narrow pouch. Opening the pouch with suturing of the bleeding point might have resulted in a significant stricture of the gastro-esophageal junction. Laparoscopic control of the left gastric artery with endoscopic identification of the bleeding vessel would have been an alternative, however, this approach would have compromised the arterial blood supply of the gastric pouch which is solely dependent upon this feeding vessel, as would angiographic embolization. For this reason, a decision was made to perform a completion gastrectomy and esophagojejunostomy. Since this patient did not undergo pre-operative EGD, and since the gastric remnant could be potential future source of bleeding, a decision was made to respect the gastric remnant as well.

Gastric bypass surgery is an effective, commonly performed procedure for morbid obesity. The approach we have adopted for massive upper GI hemorrhage in the immediate postoperative period should be distinguished from those patients who present with delayed potentially life-threatening GI bleeding after these types of operations. In these latter cases, marginal ulceration is more common than bleeding from the remnant gastric pouch, whereas determination of the site of bleeding from an excluded stomach is complex, requiring the use of novel techniques such as double-balloon enteroscopy and capsule endoscopy [12]. It is also likely that bleeding from a Dieulafoy`s lesion following gastric bypass surgery may represents a different disease when compared with other Dieulafoy`s cases, and may be triggered by sudden increase in intravascular pressure following division of the stomach. In sporadic Dieulafoy`s cases the stomach is the most common site with the majority of cases occurring along the lesser curvature. Ninety per-cent of all gastric lesions are found within $6 \mathrm{~cm}$ of the gastro-esophageal junction. One-third of the cases are extragastric, however, and may be located in the esophagus, [13] small intestine, [14] colon, [15], 
rectum [16], or the anal canal [17]. There is a higher prevalence of the Dieulafoy`s lesions in hospitalized patients with coincident cardiopulmonary dysfunction and renal impairment. Endoscopic findings typically reveal an abnormal protruding vessel within an area of normal, non-ulcerated mucosa which if bleeding at the time, shows obvious spurting. A common alternate appearance is one where adherent clot leads toward a narrow point with a miniscule mucosal defect in a region of otherwise normal mucosa.

Advances in endoscopy have resulted in an increase in the detection rates of Dieulafoy`s lesions and have expanded the options for control of hemorrhage resulting in a commensurate reduction in condition-related mortality [18]. Dieulafoy`s lesions are usually amendable to endoscopic treatment, which may include thermal electrocoagulation (with a heater or an Argon plasma probe), local injection therapy, mechanical banding or more recently, the use of novel endoscopic microsuturing technologies [19]. Overall, the risk of re-bleeding appears to be moderately high (between 10-40\%) when endoscopic monotherapy is used as compared with the utilization of a combination of endoscopic treatment options [20]. In general, angiography and embolization are reserved for endoscopically resistant cases and those patients who are sufficiently stable that they can be safely transferred to the angiography suite. In summary, a rare case of intraoperative bleeding from a Dieulafoy`s lesion is presented. This was recognized immediately following a gastric bypass procedure for morbid obesity and required an emergency total gastrectomy.

\section{Conclusion}

This is the first case of an intraoperative Dieulafoy`s lesion causing massive GI bleeding during single anastomosis gastric bypass surgery which required gastric pouch excision. Such a lesion differs from sporadic Dieulafoy`s cases, and must be considered in every case of intraoperative bleeding during gastric bypass.

\section{References}

1. Nojkov B, Cappell MS. Gastrointestinal bleeding from Dieulafoy's lesion: clinical presentation endoscopic findings and endoscopic therapy. World J Gastrointest Endosc. 2015; 7: 295-307. Ref.: https://goo.gl/13cw7z

2. Gallard T. Miliary aneurysms of the stomach, doinnant place to mortal hematemeses. Bull Soc Med Hop Paris. 1884; 1: 84-91.

3. Dieulafoy's G. Exculceratio simplex. L'intervention chirurgicale dans les hematemeses foudroyantes consecutives a l'exculceration simple de l'estomac. Bull Acad Med. 1898; 49: 49-84.

4. Braley SC, Nguyen NT, Wolfe BM. Late gastrointestinal hemorrhage after gastric bypass. Obes Surg 2002; 12: 404-407. Ref.: https://goo.gl/LoJSBk

5. Wade AD, Kothari SN. Dieulafoy`s lesion after Roux-en-Y gastric bypass. Surg Obes Rel Dis. 2009; 5 : 135-136. Ref.: https://goo.gl/3GjVEK

6. Puri $\mathrm{V}$, Alagappan $\mathrm{A}$, Rubin $\mathrm{M}$, Merola S. Management of bleeding from gastric remnant after Rouxen-Y gastric bypass. Surg Obes Relat Dis. 2012; 8: 3-5. Ref.: https://goo.gl/rC4d5R

7. Sánchez Antúnez DJ, Fernández NC, Serrano JO. Unusual case of upper gastrointestinal bleeding after laparoscopic gastric bypass: erosion of gastric remnant involving a diaphragmatic vessel. Surg Obes Relat Dis. 2011; 7: 328-329. Ref.: https://goo.gl/y8UN65

8. Lönroth H, Dalenback J, Haglind E, Lundell L. Laparoscopic gastric by-pass. Another option in bariatric surgery. Surg Endosc. 1998; 10: 636-638. Ref.: https://goo.gl/TyPBC8

9. Baettig B, Haecki W, Lammer F, Jost R. Dieulafoy's disease: endoscopic treatment and follow-up. Gut. 1993; 34: 1418-1421. Ref.: https://goo.gl/9dZUCL

10. Eisenberg D, Bell R. Intraoperative endoscopy: a requisite tool for laparoscopic resection of unusual gastrointestinal lesions--a case series. J Surg Res. 2009; 155: 318-320. Ref.: https://goo.gl/R9uFD5

11. Draper G, Layani L, MacLeish J. Management of Dieulafoy's disease with combined endoscopy and laparoscopic wedge resection. Aust N Z J Surg. 1999; 69: 156-158. Ref.: https://goo.gl/ajGXVX

12. Sakai P, Kuga R, Safatle-Ribeiro AV, Faintuch J, Gama-Rodrigues JJ, et al. Is it feasible to reach the bypassed stomach after Roux-en-Y gastric bypass for morbid obesity? The use of the double-balloon enteroscope. Endoscopy. 2005; 37: 566-569. Ref.: https://goo.gl/dVMS3Z 
13. Thimmapuram J, Sah M, Srour J. Esophageal Dieulafoy`s lesion: an unusual cause of GI bleeding. Gatrointestinal Endosc. 2011; 73: 1055-1056. Ref.: https://goo.gl/r1eq2g

14. Dulic-Lakovic E1, Dulic M, Hubner D, Fuchssteiner H, Pachofszky T, et al. Bleeding Dieulafoy`s lesions of the small bowel: a systematic study on the epidemiology and efficacy of enteroscopic treatment. Gastrointest Endosc. 2011; 74: 573-580. Ref.: https://goo.gl/kBv315

15. Jain R, Chetty R. Dieulafoy`s disease of the colon. Arch Pathol Lab Med. 2009; 133: 1865-1867. Ref.: https://goo.gl/dtYf4y

16. Yoshikumi Y, Mashima H, Suzuki J, Yamaji Y, Okamoto M, et al. A case of rectal Dieulafoy's ulcer and successful endoscopic band ligation. J Gastroenterol. 2006; 20: 287-90. Ref.: https://goo.gl/7rKcjd

17. Firat O, Karakose Y, Caliskan C, Makay O, Ozutemiz O, et al. Dieulafoy's lesion of the anal canal: report of a case. Turk J Gastroenterol. 2007; 18: 265-267. Ref.: https://goo.gl/Vi5Qgk

18. Luis LF, Sreemasimhaiah J, Jiang Tang S, Afonso BB, Rockey DC. Localization, efficacy of therapy, and outcomes of Dieulafoy`s lesions of the GI tract-the UT Southwestern GI Bleed Team experience. Gastrointest Endosc. 2008; 67: AB87.

19. Henderson JB, Sorser SA, Atia AN, Catalano MF. Repair of esophageal perforations using a novel endoscopic suturing system. Gastrointest Endosc. 2014; 80: 535-537. Ref.: https://goo.gl/zsESoe

20. Baxter M, Aly EH. Dieulafoy's lesion: current trends in diagnosis and management. Ann R Coll Surg Engl. 2010; 92: 548-554. Ref.: https://goo.gl/h8QFwB 